\title{
Cloud and IoT Based Temperature Prediction System for a Greenhouse Using Multivariate Convolutional Long Short Term Memory Network
}

\author{
Zar Zar Oo and Sabai Phyu
}

\begin{abstract}
Temperature prediction depends on various parameters to predict the dependent variables which are changing from time to time with its atmospheric variables. Predictions of Greenhouse temperature is a vital role in Greenhouse cultivation. The proposed system is collecting the climate data inside and outside of the Greenhouse by Internet of Things (IoTs) weather stations. It utilizes these historical data and provides temperature forecasts for the Greenhouse of next a few hours to a few days. In this work, the architecture of predictive weather station platform based on cloud and IoTs technology is proposed and multivariate Convolutional Long Short Term Memory Network model is used for time series forecasting. The system implementation is carried out in Vegetable and Fruit Research and Development Center (VFRDC), Ye Mon, Bago Division, Myanmar. All environmental time series weather data of the Greenhouse observed by the installed sensors and some weather information from Weather API are sent to and stored in the cloud server, ThingSpeak. The runtime for learning is provided by a cloud service, Google Colaboratory (Colab). In this work, Beijing PM2.5 dataset is analyzed by the proposed system. According to the Mean Average Error (MAE) value of the prediction result, the proposed system can predict the temperature inside the Greenhouse in advance and it can effectively promote the agricultural productivity by providing the farmers with more accurate predicted temperature, thereby minimizing the risk of temperature damage.
\end{abstract}

Index Terms-Deep learning, IoTs, LSTM, time series prediction.

\section{INTRODUCTION}

The major requirements of people can be fulfilled by the advanced modern technologies. In today's world, the Internet of Things (IoTs) technology has become a vital role in human daily routines because it can extend the perceptions and able to modify the environmental conditions. Mostly, the development of IoTs solutions is applied in the agro-industrial and environmental fields which occur in wide range of area because they need continuous monitoring and control [1]. In coming years, IoTs can become an important tool for people interacting in the agro-industrial system. Moreover, IoTs can perform environmental data collection and data storage in the cloud. Therefore, suppliers, technicians, farmers, distributors, consumers, business men, and government representatives can feed these IoTs data to the corresponding predictive models for forecasting environmental variables; thus, it can lead to faster and more

Manuscript received October 20, 2019; revised January 3, 2020.

The authors are with University of Computer Studies, Yangon, Myanmar (zarzaroo@ucsy.edu.mm). precise decisions to be a safe and healthy environment for all living things. To act as these predictive models, traditional modeling methods are insufficient to capture long-term multivariate dependencies of data for required prediction accuracy because they have complex pattern. Several deep learning models based on Recurrent Neural Network (RNN) and Convolutional Neural Network (CNN) methods have been proposed to address such concerns. But, the prediction accuracy needs to be improved and the multivariate time series data dependence for aperiodic data to be minimized.

Moreover, weather is a data-intensive, multidimensional, continuous and dynamic process because it represents the air condition for a specific place for a given period. The temperature, humidity and other weather elements at different points of location can somehow be rather different even though they are within the same area. The main aim of this work is to acquire data captured by multiple sensors and to forecast the temperature for the enhancement of the crop growth in the Greenhouse and to raise the potency and efficiency of the farmers. In this work, the focus data consists of air temperature, air humidity, soil temperature and soil moisture of the crop field and wind speed and wind direction of the field. To be these environmental factors in good conditions, farmers need to know and control the weather conditions in advance. For this reason, cloud and IoTs based Temperature Prediction System for a Greenhouse is developed by using Multivariate Convolutional Long Short Term Memory Network.

In the weather stations of the system, Arduino is used as a microcontroller and it receives input from installed sensors. Moreover, it can also control several motors in the Greenhouse, other actuators and lights. As the sensors in the weather station, DHT11 sensor, soil temperature and soil moisture sensor are used to measure temperature and humidity, the warmth in the soil, the water content in soil. As the Greenhouse components, the cooling fan, the exhaust fan, the water pump and the motor pump are also connected to and controlled by the Arduino. Installed sensors observe all environmental parameters which are sent to and stored in the cloud server. As the contribution, this work can be used to watch the factors that influence the crop yield and growth. Moreover, it is more suitable for farmers to determine what conditions, detect diseases, control machinery. The most suitable support by the proposed system is that farmers do not need to go their farms and it saves time and cost.

To predict the temperature, Multivariate Convolutional Long Short Term Memory Network is used as an automated learning tool, trained by a cloud service. A precise predictive model of temperature that can predict the 
occurrence of temperatures several hours before head to reduce financial losses. This article is organized as: Section II discusses theory background and some related works, the proposed system is presented in Section III, Section IV shows the system implementation and discussion and Section V concludes the article.

\section{BACKGROUND}

In this section, some relevant earlier researches are discussed. Then, two aspects used in the proposed system are described. The first aspect includes the IoTs technology, and the second focuses on time series predictions.

\section{A. Related Work}

In Ref. [2], Huihui $\mathrm{Yu}$ predicted the temperature in a Chinese solar Greenhouse based on Least Square Support Vector Machine optimized by improved Particle Swarm Optimization. In that work, a novel temperature prediction model based on a least squares support vector machine model with parameters optimization is proposed. The proposed model was more accurate than classical standard Support Vector Machine and Back Propagation methods.

In Ref. [3], M. Outanoute built the dynamic models for the estimations of internal temperature and relative humidity of a Greenhouse. Multilayers perceptron with 12 hidden neurons has been trained with Levenberg Marquardt algorithm which updates the weights and bias values. A hyperbolic tangent is used as an activation function. Data, several sensors data which are the outside temperature, internal temperature, external relative humidity and internal relative humidity is used as input to the model. Input-output data were collected every 10 seconds interval. The output of the model is internal temperature and humidity in the Greenhouse.

Raquel Salazar developed a Neural Network Model for five and ten minute enthalpy predictions ahead of the sensor sign based on the environmental factors inside and outside the Greenhouse in [4]. A normalized index $\beta$ was calculated for evaluating of the ventilation system. Seven Neural Network models were developed with different input variables for enthalpy prediction. 1700 data patterns are used for training, validation and testing the Neural Network models for enthalpy prediction, then the temperature and humidity in a Greenhouse was predicted.

In Ref. [5], Youjun Yue implemented a model to predict the temperature and humidity of a Greenhouse based on the Radial Basic Function network. The Levenberg-Marquardt algorithm is applied to optimize the neural network parameters. The inside and outside weather data of the Greenhouse was used as input into the system and the temperature and the humidity in a Greenhouse were predicted.

Dattatraya Shinde developed IOTS Based Environment change Monitoring and Controlling system for a Greenhouse by using Wireless Sensor Network in [6]. A simple Raspberry Pi3 based circuit watches and reads the values of environmental changes to get maximum growth of plant. The system monitors soil quality by wireless sensor nodes. Input data is acquired from each sensor and the output of the system is the temperature and the humidity in a Greenhouse.
Afan Galih Salman proposed a robust and adaptive statistical model for forecasting univariate weather variable in Indonesian airport by both single layer LSTM model and multi layers LSTM model. By using temperature, dew point, humidity and visibility from all over the world weather stations, the visibility at the airport is predicted [7]. Adding intermediate variable singal into LSTM memory block, the proposed model is extended LSTM model. Aekyeung Moon provided online frost forecasting service in [8]. Decision tree and boosted tree are used as classifiers. Real time frost data from weather stations in four regions of Yeoungcheon, South Korea is used as input and the possibility of frost in the next morning has informed to farmers in advance.

\section{B. Internet of Things (IoTs) Technology}

From the recent years, IoTs have been applied in many areas [9]. In the field of agriculture, the applications of IoTs technology are used to improve crop yields, quality and to reduce some costs. One of the applications is in precision agriculture which can assist farmers in a statistical manner by providing them make better and well informed decisions [10]. The goal of IoTs technology is to integrate sensors and actuators into everyday objects, taking advantage of the miniaturization of hardware components and minimizing economic costs. Moreover, its another goal is to connect the hardware components to the Internet via wireless and fixed networks, generate information in real time, and store it for later processing. There have been various IoTs applications in farming, such as smart agriculture and smart animal farming. In traditional farming method, farmers need to go the Greenhouse to check the humidity level, temperature level and soil moisture. This method is time consuming, labor intensive and difficult for daily work.

To solve the described problems, several sensors are used to acquire farm environmental weather parameters in the proposed system. The sensors are wired to the controller. In this work, WeMos D1 is used as a controller and is connected to the internet. WeMos D1 is a ESP8266 WiFi based board that uses the Arduino design with an operating voltage of $3.3 \mathrm{~V}$. And then, all of the collected sensor data are conveyed to the cloud for storage and future prediction.

\section{Time Series Forecasting}

Due to the nature of weather data, time series forecasting is appropriate because temperature forecasting is temporal and time series process. There are one-step univariate forecasting, multi-step or sequence forecasting and multivariate forecasting, among them, the last one is applied in the proposed system. Multivariate forecasting observes multiple different measurements and forecasts one or more of them. It includes two sets of time series observations to predict one of these or both.

\section{Long Short Term Memory (LSTM) Network Architecture}

In this work, the forecasting model is implemented by LSTM network architecture and there are some reasons for using LSTM. For example, when data sequence is long enough, Recurrent Neural Networks (RNNs) suffer from transferring information from earlier time steps to later ones. This situation, short-term memory, is the main problem of RNNs. While a paragraph of text is processing for 
predictions, RNNs may eliminate important information from the beginning.

In a back propagation neural network, there is the vanishing gradient problem. In that, gradients are used to update networks weights and the problem is occurred while gradient back propagates through time and its value converts smaller and smaller because it is multiplied by the learning rate. Therefore, it becomes extremely small after some time and it doesn't contribute too much learning.

RNNs, learning is stopped when layers get a small gradient update. If these layers are earlier time steps, they will not learn, so RNNs can forget earlier step information in longer sequences which causes a short-term memory.

LSTM has been the solutions to short-term memory of RNNs. The LSTM architecture is shown in Fig. 1.

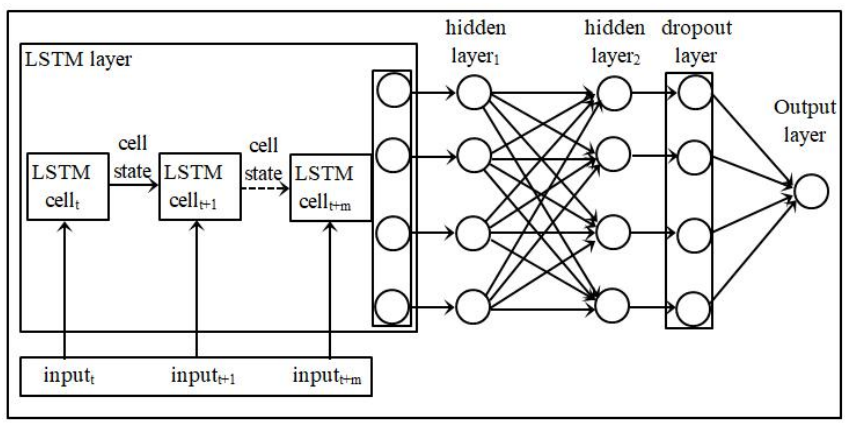

Fig. 1. The LSTM architecture.

The first concept is that the cell state transfers relative information throughout the way of the processing sequence chain and works as the memory of the network. The effect of short-term memory can be reduced because the earlier time steps information can be carried to later time steps. The second concept, the gates are different neural networks that can decide which information is allowed on the cell state. They can learn which information is needed to keep or forget during training.

There are three types of gates. They are input gate, iutput gate and forget gate. The flow of input activations into the memory cell and the output flow into the rest of the network are controlled by the input gate and the output gate respectively. The internal state of the cell is ruled by the forget gate before transferring it as input to the cell through the self-recurrent. The structure of each LSTM layer is:

$$
\begin{gathered}
\tilde{c}^{<t>}=\tanh \left(W_{c}\left[a^{<t-1>}, x^{<t>}\right]+b_{c}\right) \\
\Gamma_{u}=\sigma\left(W_{c}\left[a^{<t-1>}, x^{<t>}\right]+b_{u}\right) \\
\Gamma_{f}=\sigma\left(W_{f}\left[a^{<t-1>}, x^{<t>}\right]+b_{f}\right) \\
\Gamma_{o}=\sigma\left(W_{o}\left[a^{<t-1>}, x^{<t>}\right]+b_{o}\right) \\
\left.c^{<t>}=\Gamma_{u} * \tilde{c}^{<t>}+\Gamma_{f} * c^{<t-1>}\right) \\
a^{<t>}=\Gamma_{o} * \tanh c^{<t>}
\end{gathered}
$$

In equations (1-6), $x^{<t>}$ is the input vector of the LSTM unit. $\Gamma_{u}$ is the update gate's activation vector. $\Gamma_{f}$ is the forget gate's activation vector. $\Gamma_{o}$ is the output gate's activation vector. $W_{c}, b_{c}, W_{u}, b_{u}, W_{f}, b_{f}, W_{o}$ and $b_{o}$ are the trainable parameters. $c^{<t-1>}$ is the memory from last LSTM unit. $c^{<t>}$ is the new updated memory. $a^{<t-1>}$ is the output of last LSTM unit and $a^{<t>}$ is the current output. Therefore, LSTM can perform connection of the cell, forgetting or resetting the cell's memory as shown in Fig. 2.

The proposed system is extended to LSTM by integrating the Convolutional Neural Network (CNN) layers with LSTM for extracting significant features from the sensor data input. When data passes through Convolutional Layers, several filters extract important features. After passing these layers, the output is connected to a LSTM network layers. The predicted weather station platform is proposed in the next session.

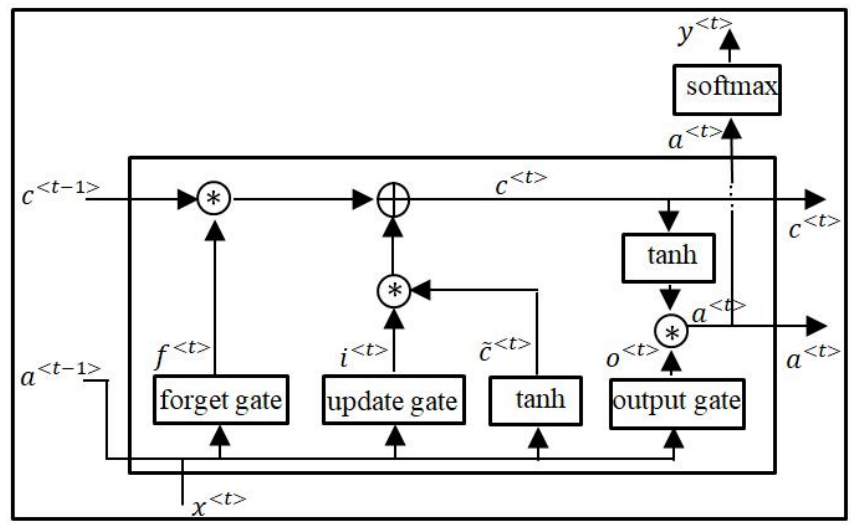

Fig. 2. The LSTM cell.

\section{The Proposed Predictive Weather Station PLATFORM}

The proposed predictive weather station platform, shown in the Fig. 3, collects microclimate information inside and outside the Greenhouse and global weather information from the web, WeatherAPI. Now, all data from the sensors and the web are collected every five minutes in the research field, VFRDC and stored in the cloud database. To build the predictive model, the Greenhouse outside data and web data are used as history data(input data) and Greenhouse inside data is used as target value(forecast temperature). The stored history data are downloaded to Google Colab cloud and preprocessed. The preprocessed time series data are converted to a supervised learning problem by framing of time series problem given the desired length of input and output sequences. A supervised learning problem is included input patterns (X) and output patterns (y). Therefore, machine learning algorithms can learn how to forecast the output patterns from the input patterns. By using these pairs of input and output sequences, the model is trained by LSTM network architecture with CNN layer. The temperature value predicted by the model is passed to the farmers in advance. System requirements for the proposed system are described in the next section.

\section{IMPLEMENTATION}

Some system requirements are described in this section. Electronic devices of the weather stations are kept in a waterproof box and it is regarded as the control box for the system. The control box is placed in the Greenhouse, having DHT11 sensor as shown in Fig. 4. For the outside weather station, DHT11 sensor, the soil moisture sensor and soil temperature sensor are connected to the microcontroller, WeMos D1 as shown in Fig. 5. 


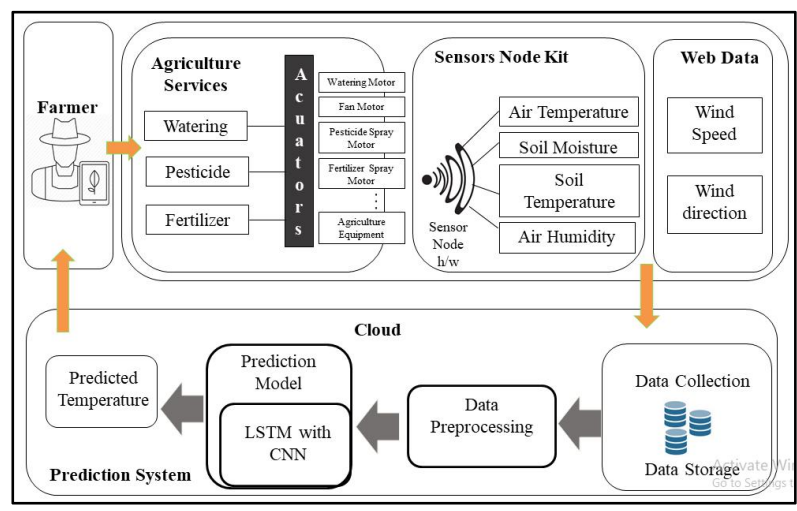

Fig. 3. The proposed predictive weather station platform.

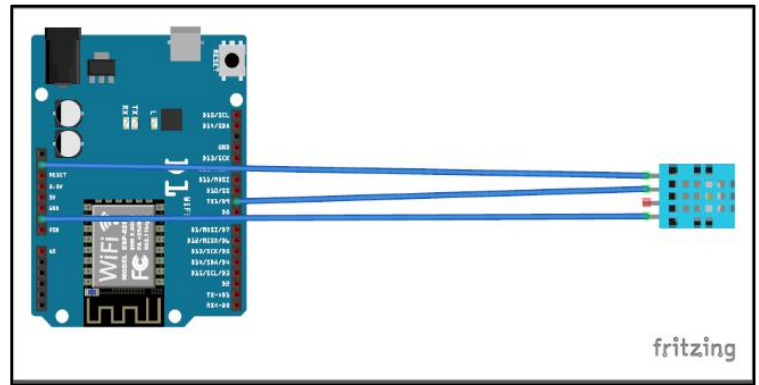

Fig. 4. Greenhouse inside weather station.

In this work, as an IoTs technology, the soil moisture sensor and soil temperature sensor measure the humidity and temperature of soil and WeMos D1 controls the switching on and off of water sprinklers by farmer's decision. The solenoid valve was used to control water flow with on/off action. The DHT11 sensor was used to measure the temperature and humidity of the farm.

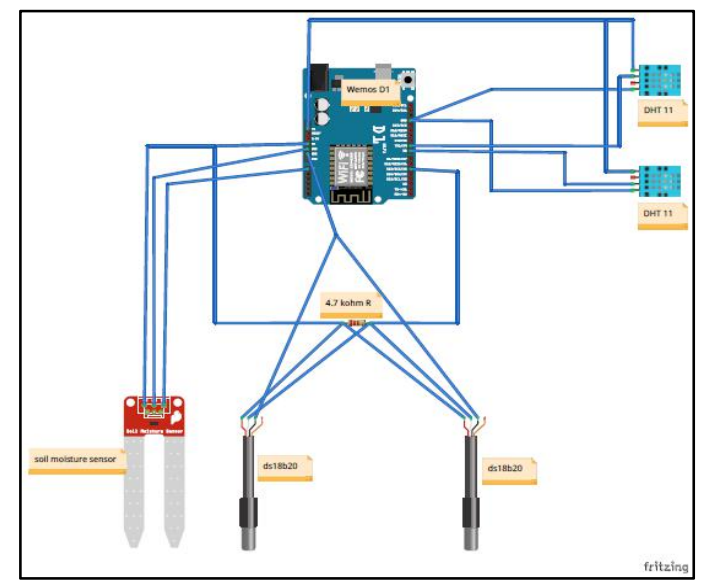

Fig. 5. Greenhouse outside weather station

The second part is IoTs analytic platform, ThingSpeak gets agricultural information from the microcontroller, Wemos D1 which accesses the internet via mobile data. The agricultural information from the sensors and wind speed and wind direction from the Weather API are also collected in the ThingSpeak cloud. ThingSpeak also displays the details field information and weather conditions by the specific channels.

Farmers can watch the inside and outside weather conditions of the Greenhouse as shown in the Fig. 6-Fig. 11. Fig. 6 and Fig. 7 display temperature and humidity outside the Greenhouse. Fig. 8 shows Greenhouse outside soil temperature. Greenhouse outside soil moisture is plotted in
Fig. 9, Fig. 10, and Fig. 11 display Greenhouse Inside temperature and humidity.

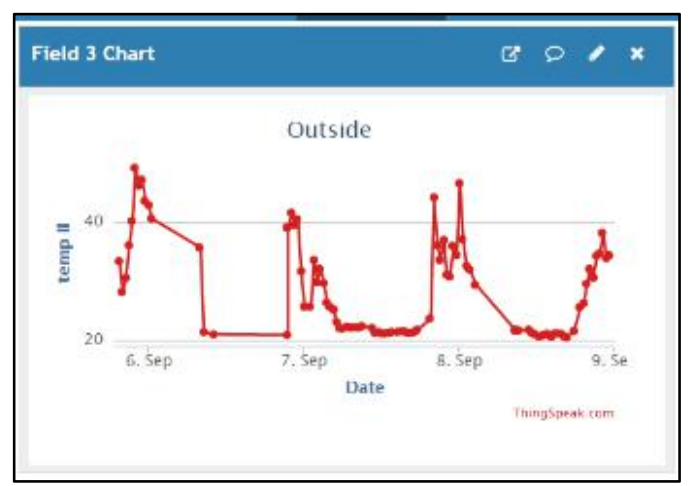

Fig. 6. Greenhouse outside temperature.

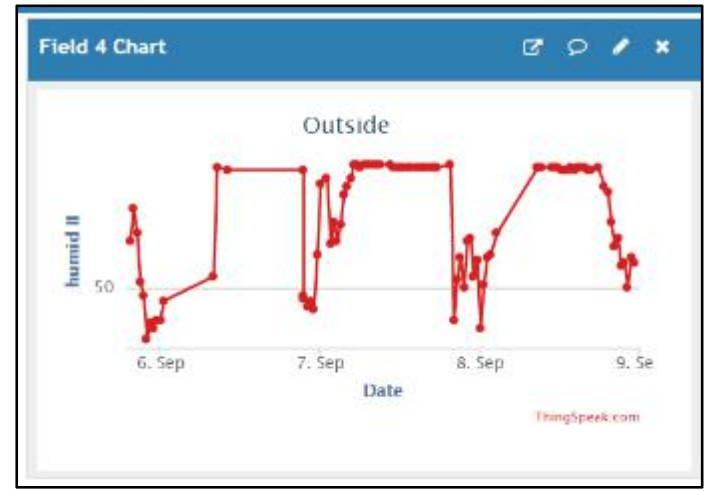

Fig. 7. Greenhouse outside humidity.

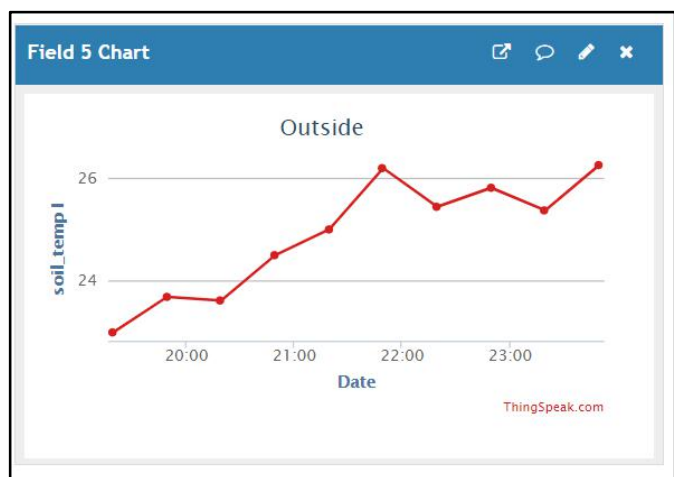

Fig. 8. Greenhouse outside soil temperature.

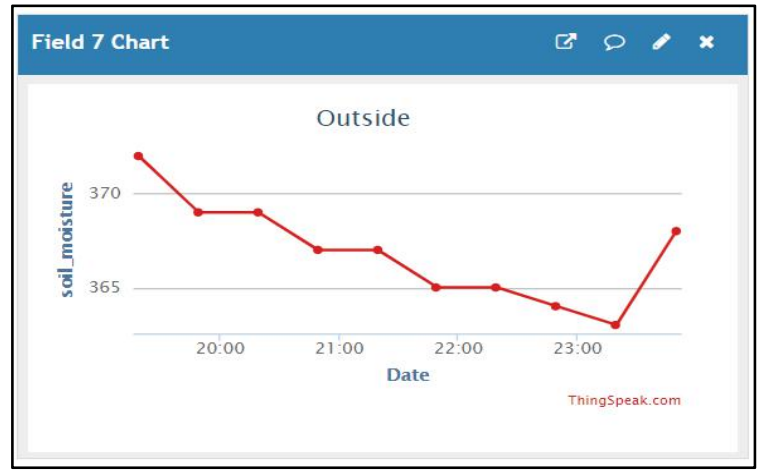

Fig. 9. Greenhouse outside soil moisture.

The proposed system will also forecast the predicted temperature in 12 hours advance by using multivariate time series prediction. As a cloud service, Google Colaboratory(Colab) provides a runtime for deep learning and is used to train the network.

The final part is the mobile application which was 
implemented for interfacing with the farmer. This part is used to observe the weather conditions at any time and the farmer can control on-off switching of the electrical system.

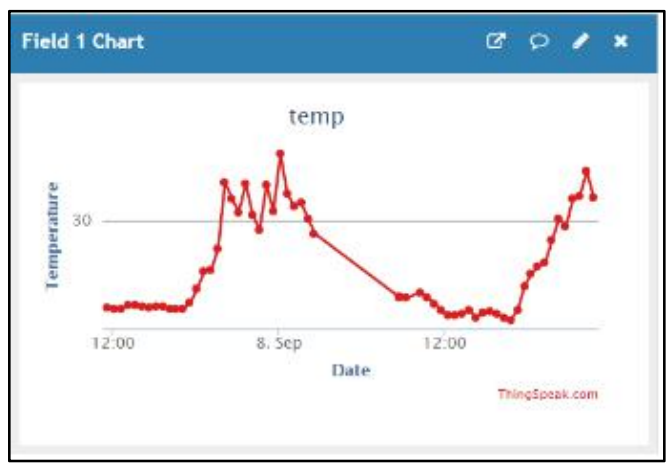

Fig. 10. Greenhouse inside temperature.

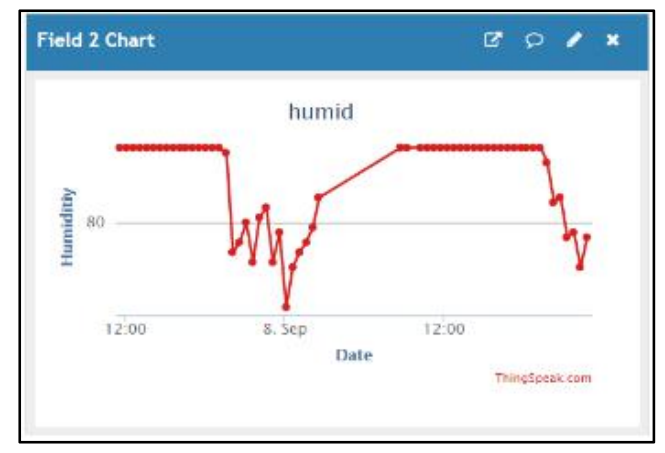

Fig. 11. Greenhouse inside humidity.

\section{EXPERIMENTS}

\section{A. Data Acquisition}

Weather data of the Greenhouse for the proposed system are collecting now in Vegetable and Fruit Research and Development Center (VFRDC), Ye Mon, Bago Division, Myanmar. Inside the Greenhouse, the mainly physical influencing factors on the temperature mainly contain the outside temperature, air humidity, soil temperature, soil moisture, wind speed and wind direction. Therefore, the training set for the model is established as in Fig. 12.

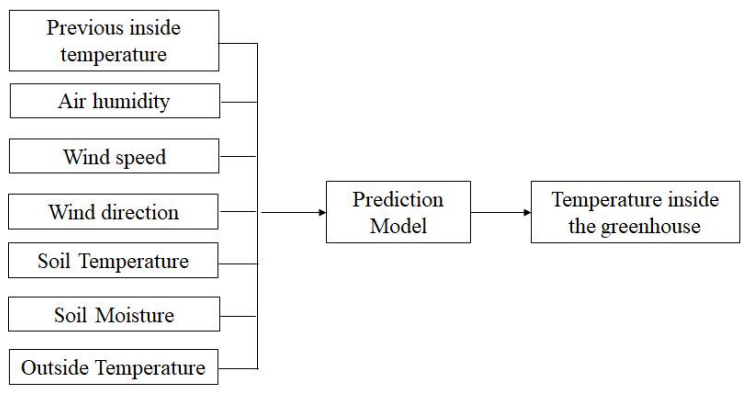

Fig. 12. Establishing the training sets of the model.

Data from the research field need some time to get the amount enough for training the network. Therefore, nine years period of temperature time series data Beijing PM2.5 dataset shown in Fig. 13 is used as a case study to build the model of the proposed system. The entire data set (with 420551 data samples) for every 10 minutes of 9 years weather data into three splits as training, validation and testing set. The first 30000 data samples are considered as training data split.

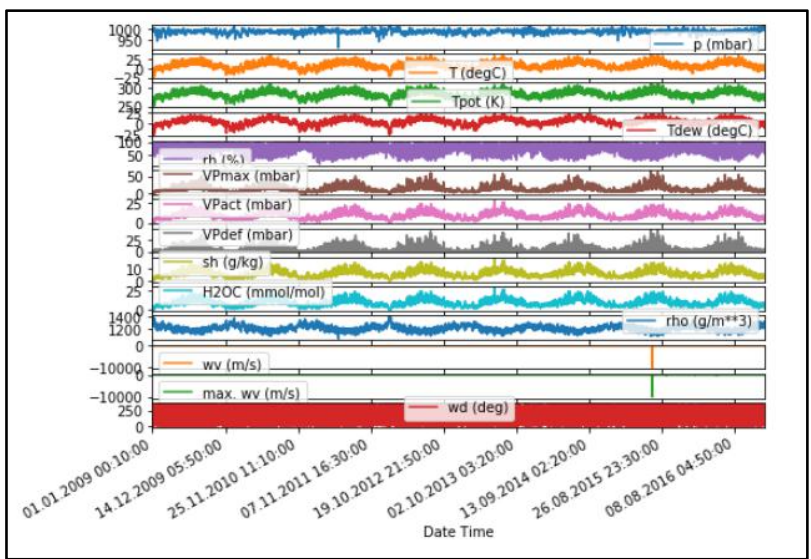

Fig. 13. Plotting time series data(Beijing PM2.5 dataset).

\section{B. Data Normalization}

All of data splits were scaled to values between 0 and 1 using the eq(7) because the sample span is large.

$$
x=\frac{x-\operatorname{mean}(x)}{\max (x)-\min (x)}
$$

To build the training dataset, five days time series data is collected as input features and next twelve hours data as target forecast value.

\section{Experimental Results}

This section provides the analysis of experimental results for the proposed model. To evaluate the learning model, Mean Absolute Error (MAE) in eq(8) is used.

$$
M A E=\sum_{i=0}^{n} \frac{y i-\overline{y i}}{n}
$$

The following table describes the network training model for the experiment. After data normalization, the preprocessed sensor data are passed through Convolutional layer which extract important features. After convolutional layer, data are conveyed into LSTM layers. Then, the output is connected to a fully-connected dense network. The last layer, Lamda layer is used for dynamically changing of learning to achieve the minimum loss.

\begin{tabular}{c|c|c}
\multicolumn{3}{c}{ TABLE I: TRAINING MODEL } \\
\hline \hline Layer (type) & Output Shape & Param \# \\
\hline conv1d_2 (Conv1D) & (None, 120, 32) & 2272 \\
\hline lstm_4 (LSTM) & (None, 120, 64) & 24832 \\
\hline lstm_5(LSTM) & (None, 120, 64) & 33024 \\
\hline dense_6 (Dense) & (None, 120, 30) & 1950 \\
\hline dense_7 (Dense) & (None, 120, 10) & 310 \\
\hline dense_8 (Dense) & (None, 120, 1) & 11 \\
\hline lambda_2 (Lambda) & (None, 120, 1) & 0 \\
\hline \hline
\end{tabular}

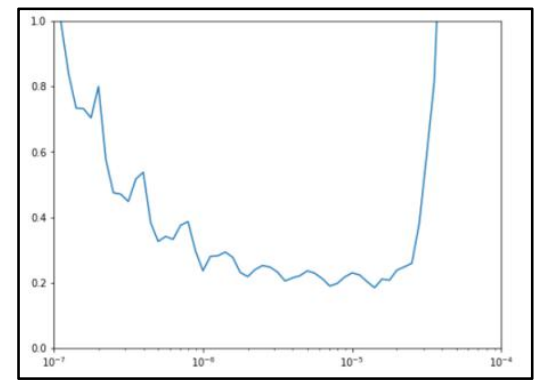

Fig. 14. Loss with learning rate during training.

During network is being trained by evaluation interval 
200, the relationship between the loss and the learning rate is presented in Fig. 14. In the figure, the $\mathrm{X}$-axis represents the learning rate and $\mathrm{Y}$-axis represents the loss. The minimum loss can be got by adjusting the learning rate. By the graph, the minimum loss is reached when the learning rate is $1 \mathrm{e}-5$.

\section{CONCLUSION}

IoTs technology has been applied in agriculture for the improvement of crop yields and quality and to reduce costs. For these facts, cloud and IoTs based temperature prediction system for a Greenhouse was proposed. A system to monitor and control environmental factors near the crop fields was designed and implemented in this paper. For non-linear forecasting model, Multivariate Convolutional Long Short Term Memory Network model is proved to be an effective model of the temperature forecast decision. The experimental results indicate that the proposed model has a high accuracy and efficiency for prediction. When farmers know the weather warnings in advance, they can act valuable added services and prevention plan for the crops. Thus, the proposed model provides a good example of the capabilities of Convolutional LSTM network model for modeling high precision and efficient temperature forecasting.

\section{CONFLICT OF INTEREST}

The authors declare no conflict of interest.

\section{AUTHOR CONTRIBUTIONS}

Z. Z. O. and S. P. conceived and designed the experiments; Z. Z.O. and S. P. performed the experiments; Z. Z. O. wrote the paper.

\section{ACKNOWLEDGEMENT}

The authors would like to thank all of their teachers in their lives, their families and their friends.

\section{REFERENCES}

[1] J. Muangprathub, N. Boonnam, S. Kajornkasirat, N. Lekbangpong, A. Wanichsombat, and P. Nillaor, "IoTs and agriculture data analysis for smart fan,” Elsevier, June 2018, pp. 467-474.
[2] H. Yu, Y. Chen, S. G. Hassan, and D. Li, "Prediction of the temperature in a chinese solar Greenhouse based on LSSVM optimized by improved PSO," Elsevier, pp. 94-102, January 2016.

[3] M. Outanoute, A. Lachhab, A. Ed-dahhak, A. Selmani, M. Guerbaoui, and B. Bouchikhi, "A neural network dynamic model for temperature and relative humidity control under Greenhouse," in Proc. IEEE Publishing in 2015 Third International Workshop on RFID And Adaptive Wireless Sensor Networks(RAWSN), May 13-15, 2015, pp. 6-11.

[4] R. Salazar, A. Rojano, Irineo, and U. Schmidt, "A model for the combine description of the temperature and relative humidity regime in the greenhouse," in Proc. IEEE Ninth Mexican International Conference on Artificial Intelligence, Nov 8-13, 2010, pp. 114-117.

[5] Y. Yue, J. Quan, H. Zhao, and H. Wang, "The prediction of greenhouse temperature and humidity based on LM-RBF network," in Proc. 2018 IEEE International Conference on Mechatronics and Automation, August 5-8, 2018, pp. 1537-1541.

[6] D. Shinde and N. Siddiqui, "IOTS based environment change monitoring and controlling in greenhouse using WSN," in Proc. 2018 International Conference on Information, Communication, Engineering and Technology (ICICET), IEEE, Aug. 29-31, 2018.

[7] A. G. Salman, Y. Heryadi, E. Abdurahman, and W. Suparta, " Single layer \& multi-layer long short-term memory (LSTM) model with intermediate variables for weather forecasting," in Proc. $3^{\text {rd }}$ International Conference on Computer Science and Computational Intelligence, 2018, pp. 89-98

[8] A. Moon, K. Y. Moon, S. W. Son, Microclimate-Based Predictive Weather Station Platform: A Case Study for Frost Forecast, 2017.

[9] T. Ojha, S. Misra, and N. S. Raghuwanshi, "Wireless sensor networks for agriculture: The state-of-the-art in practice and future challenges," Computers and Electronics in Agriculture, vol. 118, pp. 66-84, October 2015.

[10] R. K. Kodali, N. Rawat, L. Boppana, "WSN sensors for precision agriculture," in Proc. IEEE Region 10 Symposium, April 2014.

Copyright (C) 2020 by the authors. This is an open access article distributed under the Creative Commons Attribution License which permits unrestricted use, distribution, and reproduction in any medium, provided the original work is properly cited (CC BY 4.0).

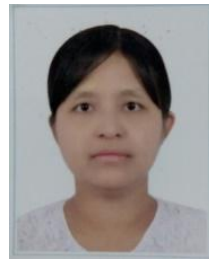

Zar Zar Oo is a $\mathrm{PhD}$ candidate at the University of Computer Studies, Yangon. She is very interested in IoTs technology, machine learning and computing. Her current research is related to cloud and IoT based technology and time series forecasting.

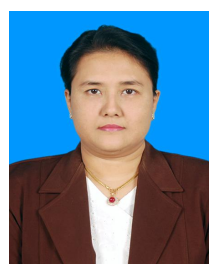

Sabai Phyu is a dean of Cloud Computing Lab at the University of Computer Studies, Yangon (UCSY). She is interested in computer science, information theory and cloud computing. Her research areas include IoTs, cloud computing and time series forecasting. She currently works at the Software Department, UCSY. 